PROCEEDINGS OF THE

AMERICAN MATHEMATICAL SOCIETY

Volume 137, Number 3, March 2009, Pages 1153-1160

S 0002-9939(08)09604-4

Article electronically published on September 29, 2008

\title{
ACCIDENTAL PARABOLICS IN THE MAPPING CLASS GROUP
}

\author{
CHRISTOPHER J. LEININGER
}

(Communicated by Alexander N. Dranishnikov)

\begin{abstract}
In this paper we discuss the behavior of the Gromov boundaries and limit sets for the surface subgroups of the mapping class group with accidental parabolics constructed by the author and A. Reid (2006). Specifically, we show that generically there are no Cannon-Thurston maps from the Gromov boundary to Thurston's boundary of Teichmüller space.
\end{abstract}

\section{INTRODUCTION}

Let $S=S_{g}$ be a closed genus $g$ surface $(g \geq 2)$ and $\operatorname{Mod}(S)$ its mapping class group. In [8], we consider a family of subgroups $G\left(\omega_{g}\right)<\operatorname{Mod}\left(S_{g}\right)$ depending on a certain holomorphic abelian differential $\omega_{g}$ constructed by Veech [14, 15] (we recall the relevant geometry of these groups below). The group $G\left(\omega_{g}\right)$ is free on $2 g$-generators but is naturally isomorphic to the fundamental group of a surface of genus $g$ with one puncture. Indeed, $G\left(\omega_{g}\right)$ stabilizes a totally geodesic hyperbolic plane $\mathbb{H}_{\omega_{g}}$ in Teichmüller space with quotient a one-cusped hyperbolic surface. We write $G_{0}\left(\omega_{g}\right)<G\left(\omega_{g}\right)$ for a representative of the unique conjugacy class of maximal parabolic subgroups.

For each $g$, we can abstractly double $G\left(\omega_{g}\right)$ over $G_{0}\left(\omega_{g}\right)$ to obtain $\mathcal{G}_{2 g}$, the fundamental group of a closed surface of genus $2 g$,

$$
\mathcal{G}_{2 g}=G\left(\omega_{g}\right) *_{G_{0}\left(\omega_{g}\right)} G\left(\omega_{g}\right) .
$$

We can attempt to carry out this doubling within $\operatorname{Mod}(S)$ by conjugating one of the factors of $\mathcal{G}_{2 g}$ by an element of $\mathrm{C}\left(G_{0}\left(\omega_{g}\right)\right)$, the centralizer of $G_{0}\left(\omega_{g}\right)$ in $\operatorname{Mod}(S)$. More precisely, for every $h \in \mathrm{C}\left(G_{0}\left(\omega_{g}\right)\right)$ and $n \in \mathbb{Z}$ we obtain a homomorphism from $\mathcal{G}_{2 g}$ to the subgroup of $\operatorname{Mod}(S)$ generated by $G\left(\omega_{g}\right)$ and its conjugate by $h^{n}$ :

$$
\mathcal{G}_{2 g} \rightarrow \mathcal{G}\left(\omega_{g}, h, n\right)=\left\langle G\left(\omega_{g}\right), h^{n} G\left(\omega_{g}\right) h^{-n}\right\rangle<\operatorname{Mod}(S) .
$$

This homomorphism is the canonical one extending the obvious isomorphisms of the first and second factors to $G\left(\omega_{g}\right)$ and $h^{n} G\left(\omega_{g}\right) h^{-n}$, respectively.

For arbitrary $h \in \mathrm{C}\left(G_{0}\left(\omega_{g}\right)\right)$ and $n \in \mathbb{Z}$ this homomorphism need not be injective. This setup however is reminiscent of the Maskit Combination Theorem in hyperbolic geometry [9]. In [8, we show that under mild hypotheses, one is able to make a similar conclusion to that of Maskit's Theorem. To state the result precisely, we recall that $G_{0}\left(\omega_{g}\right)$ is (virtually) generated by a multitwist in a multicurve $A$ (see Section 2).

Received by the editors January 23, 2008, and, in revised form, April 8, 2008 2000 Mathematics Subject Classification. Primary 57M60; Secondary 30F60. Research supported by NSF DMS 06-03881.

(C)2008 American Mathematical Society 1153

Reverts to public domain 28 years from publication 
Theorem 1.1 (Leininger-Reid [8]). Suppose $h \in \mathrm{C}\left(G_{0}\left(\omega_{g}\right)\right)$ is pseudo-Anosov on $S-A$. Then

$$
\mathcal{G}_{2 g} \rightarrow \mathcal{G}\left(\omega_{g}, h, n\right)<\operatorname{Mod}\left(S_{g}\right)
$$

is an isomorphism for all sufficiently large $n$. Moreover, every element of $\mathcal{G}\left(\omega_{g}, h, n\right)$ is pseudo-Anosov except those conjugate into $G_{0}\left(\omega_{g}\right)$.

The proof of Theorem 1.1 in [8] mimics the proof of Maskit's Theorem, at least in principle, and the groups $\mathcal{G}\left(\omega_{g}, h, n\right)$ are analogous to Kleinian surface groups with an accidental parabolic. One may wonder the extent to which these groups behave like their Kleinian counterparts. For example, one can ask if they satisfy some form of geometric finiteness; see Problem 6.1 of [12. One ingredient that seems desirable for such a notion is a description of the ideal boundary behavior; compare Floyd [3] for the Kleinian setting. In [8, Question 10.1], we ask whether or not there is a Cannon-Thurston map for these groups,

$$
\partial \mathcal{G}\left(\omega_{g}, h, n\right) \rightarrow \mathbb{P} \mathcal{M L}\left(S_{g}\right) .
$$

By this, we mean a $\mathcal{G}\left(\omega_{g}, h, n\right)$-equivariant continuous map from the Gromov boundary of $\mathcal{G}\left(\omega_{g}, h, n\right)$ to Thurston's boundary of Teichmüller space, $\mathbb{P} \mathcal{M L}\left(S_{g}\right)$.

For the factor subgroups $G\left(\omega_{g}\right), h^{n} G\left(\omega_{g}\right) h^{-n}$ and all their conjugates one does have Cannon-Thurston maps in this sense. Moreover, when a pair of these subgroups nontrivially intersect, the Cannon-Thurston maps agree on the boundary of the common subgroup. Thus one can begin to build a "finite approximation" to the Cannon-Thurston map for the amalgam using these as building blocks. Despite the existence of this approximation, we show that in general there is no such map.

Theorem 1.2. For all $g \geq 2$, "generic" $h$ as in Theorem 1.1 and $n$ sufficiently large, there does not exist a Cannon-Thurston map

$$
\partial \mathcal{G}\left(\omega_{g}, h, n\right) \rightarrow \mathbb{P} \mathcal{M L}\left(S_{g}\right) .
$$

Here "generic" means that the stable lamination of $h$ lies outside a particular closed positive codimension subset of the appropriate space of projective measured laminations; see Section 2 for a precise definition. In genus 2 for example this subset consists of just a finite set of points, and so generic means not one of a finite set of choices, up to powers and Dehn twists in the components of $A$.

Remark 1.3. In [12] Mosher considers a different boundary for $\mathcal{G}\left(\omega_{g}, h, n\right)$, viewing $\mathcal{G}\left(\omega_{g}, h, n\right)$ (more naturally) as a relatively hyperbolic group. Then he asks about the existence of a Cannon-Thurston map to $\mathbb{P} \mathcal{M L}(S)$ from this boundary. Since this boundary is a $\mathcal{G}\left(\omega_{g}, h, n\right)$-equivariant quotient of the Gromov boundary, Theorem 1.2 also shows that generically no Cannon-Thurston map from this boundary exists either.

We also point out that the notion of a Cannon-Thurston map we consider here is weaker than that considered in the Kleinian setting. There a Cannon-Thurston map is an extension of a continuous equivariant map $G \rightarrow \mathbb{H}^{3}$ to a continuous equivariant map $\bar{G} \rightarrow \overline{\mathbb{H}}^{3}$. We have removed any discussion of the behavior in Teichmüller space and the interface with the boundary where the behavior is known to be bad; see [10. Since our notion is weaker, and since we are proving a negative result, we note that Theorem 1.2 would also hold if we were to use the stronger definition. 
Whether or not there is ever a Cannon-Thurston map for a group constructed from Theorem 1.1 remains an open question. However, from the proof it should be clear that such an example, if it exists, would have to be very special.

The obstruction to extending the finite approximation to an actual CannonThurston map lies in the action of parabolic mapping classes on Thurston's boundary $\mathbb{P} \mathcal{M L}(S)$. In hyperbolic geometry a parabolic isometry fixes a unique point on the boundary of hyperbolic space and every other point is attracted to that point under iteration. As is well known, this is not the case for the mapping class group where there is an entire simplex of fixed points, each one "equally attractive" (compare Lemma 4.1 below).

We mention that if we forget the transverse measures, this simplex of attractors collapses to a point. This collapse erases the problem in building the CannonThurston map, and in [6] we prove the following.

Theorem 1.4 (6]). For all $g \geq 2$, all $h$ as in Theorem 1.1 and all $n$ sufficiently large, there exists a continuous $\mathcal{G}\left(\omega_{g}, h, n\right)$-equivariant map

$$
\partial \mathcal{G}\left(\omega_{g}, h, n\right) \rightarrow \mathcal{G} \mathcal{L}(S),
$$

where $\mathcal{G} \mathcal{L}(S)$ is the space of geodesic laminations with the Thurston topology.

This is just one part of a more general investigation into the geometry of the groups constructed in [8], as well as a generalization to a class of groups we call graphs of Veech groups.

\section{The EXAMPles REVisited}

For every $g \geq 2$, Veech constructed a genus $g$ Riemann surface together with an abelian differential $\omega_{g}$ by appropriately gluing together a pair of regular $(2 g+1)$-gons [14, 15. This determines a singular Euclidean structure and an associated affine group Aff $^{+}\left(\omega_{g}\right)$. Locally integrating $\omega_{g}$ produces preferred coordinates around all nonzero points of $\omega_{g}$ for which the transition functions are translations. The derivative in these preferred coordinates defines a homomorphism

$$
D: \operatorname{Aff}^{+}\left(\omega_{g}\right) \rightarrow \mathrm{SL}_{2}(\mathbb{R}) \text {. }
$$

The image of this map is denoted by $\mathrm{SL}\left(\omega_{g}\right)$. The quotient by the center is $\operatorname{PSL}\left(\omega_{g}\right)<\operatorname{PSL}_{2}(\mathbb{R})$ and is a Fuchsian triangle group of type $(2,2 g+1, \infty)$.

The homomorphism $D$ is actually an isomorphism onto $\operatorname{SL}\left(\omega_{g}\right)$ (compare [7, Section 7$)$. The quotient $\operatorname{Aff}^{+}\left(\omega_{g}\right) \rightarrow \operatorname{PSL}\left(\omega_{g}\right)$ has a central kernel of order two generated by a hyperelliptic involution we denote by $\sigma$.

The commutator subgroup of $\operatorname{PSL}\left(\omega_{g}\right)$ is a free Fuchsian group with genus $g$ and one cusp. As this is free, we can choose a lift to a free subgroup of $\operatorname{Aff}^{+}\left(\omega_{g}\right)$ and this is the subgroup we denote by $G\left(\omega_{g}\right)<\operatorname{Aff}^{+}\left(\omega_{g}\right)$.

There is a pair of cylinder decompositions for $\omega_{g}$, the core curves of which are multicurves

$$
A=a_{1} \cup \ldots \cup a_{g} \quad \text { and } \quad B=b_{1} \cup \ldots \cup b_{g} .
$$

These multicurves form a "chain" on $S$ as illustrated in Figure 1. The Dehn twists in the components of each multicurve compose to multitwists

$$
T_{A}=T_{a_{1}} \circ \ldots \circ T_{a_{g}} \quad \text { and } \quad T_{B}=T_{b_{1}} \circ \ldots \circ T_{b_{g}}
$$

and $T_{A}, T_{B} \in \mathrm{Aff}^{+}\left(\omega_{g}\right)$ with $D\left(T_{A}\right)$ and $D\left(T_{B}\right)$ parabolic. We note that $\sigma$ is the hyperelliptic involution of $S$ leaving each of the components of $A$ and $B$ invariant. 


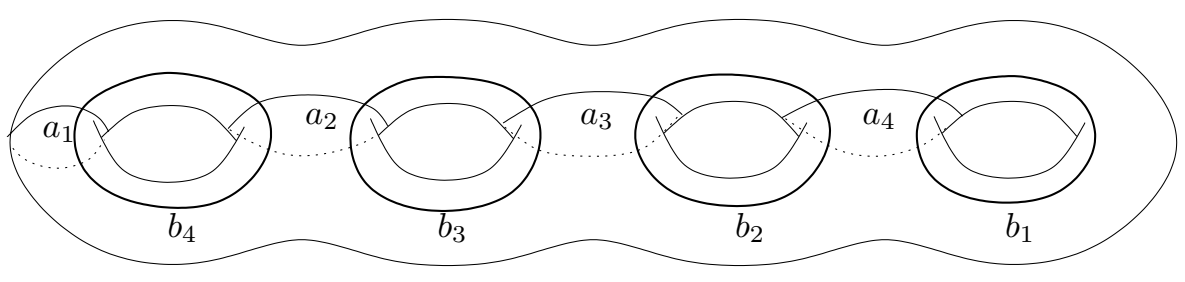

Figure 1. $A=a_{1} \cup \ldots \cup a_{4}$ and $B=b_{1} \cup \ldots \cup b_{4}$ in genus 4 .

Since $\operatorname{Aff}^{+}\left(\omega_{g}\right)$ has one conjugacy class of parabolic subgroups, $T_{A}$ and $T_{B}$ are conjugate in $\mathrm{Aff}^{+}\left(\omega_{g}\right)$. Similarly, the one conjugacy class of parabolic subgroups of $G\left(\omega_{g}\right)$ is represented by a cyclic subgroup $G_{0}\left(\omega_{g}\right)<G\left(\omega_{g}\right)$ which contains a power of $T_{A}$. More precisely

$$
G_{0}\left(\omega_{g}\right)=\left\langle T_{A}^{2(2 g+1)} \sigma\right\rangle .
$$

Now consider $h \in \mathrm{C}\left(G_{0}\left(\omega_{g}\right)\right)$. As $h$ centralizes $T_{A}^{4(2 g+1)}=\left(T_{A}^{2(2 g+1)} \sigma\right)^{2}$, it follows that it must leave $A$ invariant. Therefore it restricts to a mapping class on $S-A$. As was stated in Theorem 1.1. we assume that this restriction of $h$ is pseudo-Anosov. We note that $h \in \mathrm{C}\left(G_{0}\left(\omega_{g}\right)\right)$ if and only if it leaves $A$ invariant and commutes with $\sigma$.

Remark 2.1. In [8] we erroneously stated that $G_{0}\left(\omega_{g}\right)$ was generated by a power of $T_{A}$, instead of by $T_{A}^{2(2 g+1)} \sigma$. The reason that the generator is $T_{A}^{2(2 g+1)} \sigma$ instead of $T_{A}^{2(2 g+1)}$ is that for any torsion-free 1-cusped Fuchsian group of finite area, when lifted to a subgroup of $\mathrm{SL}_{2}(\mathbb{R})$ the generator of the parabolic subgroup must have trace $=-2$, and not +2 ; see [1] for a nice discussion. This only affects the possible choices of $h$, requiring that it leave $A$ fixed and also commute with $\sigma$.

We now explain the meaning of "generic" in the statement of Theorem 1.2. The elements $h \in \mathrm{C}\left(G_{0}\left(\omega_{g}\right)\right)$ are described (up to twists in components of $A$ ) as pseudo-Anosov mapping classes $\hat{h}$ of $S-A$ which commute with the hyperelliptic $\hat{\sigma}=\left.\sigma\right|_{S-A}$. Let $X \subset \mathbb{P} \mathcal{M L}(S-A)$ denote the fixed points set of $\hat{\sigma}$ in $\mathbb{P} \mathcal{M L}(S-A)$. The space $X$ is alternatively described as the lifts of projective classes of laminations on $(S-A) /\langle\hat{\sigma}\rangle$, and so $X \cong S^{2 g-3}$. Further note that the set of all fixed points of the pseudo-Anosov mapping class $\hat{h}$ of $S-A$ commuting with $\hat{\sigma}$ is dense in $X$ since the same is true of the pseudo-Anosov fixed points in $\mathbb{P} \mathcal{M L}((S-A) /\langle\hat{\sigma}\rangle)$. We will construct a closed, positive codimension subset $Y \subset X$, and say that $h$ is generic if the stable fixed point of $\hat{h}$ lies outside of $Y$.

\section{LIMIT SETS AND PARABOLIC FIXED POINTS}

The limit set for a nonelementary subgroup $G<\operatorname{Aff}^{+}(\omega)$ of the affine group of an abelian differential $\omega$ has a fairly concrete description. For this, note that the set of projective classes of vertical foliations for complex multiples of $\omega$, which we denote by $\mathbb{P} \mathcal{M L}(\omega)$, is a circle in $\mathbb{P} \mathcal{M L}(S)$. In fact, the associated Teichmüller disk has an ideal boundary $\partial \mathbb{H}_{\omega}$, which by the isometry $\mathbb{H}^{2} \cong \mathbb{H}_{\omega}$, naturally admits a projective structure $\partial \mathbb{H}_{\omega} \cong \mathbb{R P}^{1}$. Moreover, there is a natural $\mathrm{Aff}^{+}(\omega)$-equivariant, piecewise projective homeomorphism $\partial \mathbb{H}_{\omega} \rightarrow \mathbb{P} \mathcal{M L}(\omega)$; see [5], Theorem 2.1. The point is 
that a ray in $\mathbb{H}_{\omega}$ comes from a 1-parameter family of Teichmüller deformations where the measure on the vertical foliation of some complex multiple of $\omega$ tends to zero. We caution the reader that according to the work of Masur [10, the map $\partial \mathbb{H}_{\omega} \rightarrow \mathbb{P} \mathcal{M L}(\omega)$ is not in general the continuous extension of the embedding of $\mathbb{H}_{q}$ into Teichmüller space.

Because a nonelementary subgroup $G<\mathrm{Aff}^{+}(\omega)$ contains a pseudo-Anosov mapping class, it follows from the work of McCarthy and Papadopoulos [11] that its limit set $\Lambda_{G}$ is the unique minimal closed $G$-invariant set. Since $\mathbb{P} \mathcal{M L}(\omega)$ is a closed $\operatorname{Aff}^{+}(\omega)$-invariant set, we see that $\Lambda_{G} \subset \mathbb{P} \mathcal{M L}(\omega)$. By minimality of limit sets for Fuchsian groups, $\Lambda_{G}$ is precisely the image of the Fuchsian limit set $\Lambda_{D(G)} \subset \partial \mathbb{H}_{\omega}$. In particular when $G$ is a Veech group - that is, a lattice such as $G\left(\omega_{g}\right)$ - the limit set is precisely $\Lambda_{G}=\mathbb{P} \mathcal{M L}(\omega)$.

We denote the foliations of $\omega_{g}$ associated to the cylinder decompositions discussed above by $\nu_{A}$ and $\nu_{B}$, respectively. The projective classes $\left[\nu_{A}\right],\left[\nu_{B}\right] \in$ $\mathbb{P} \mathcal{M L}\left(\omega_{g}\right)$ lie in the simplex of measures in $\mathbb{P} \mathcal{M L}(S)$ determined by $A$ and $B$, respectively. Note that $\left[\nu_{A}\right]$ (respectively $\left.\left[\nu_{B}\right]\right)$ is the unique fixed point in $\mathbb{P} \mathcal{M L}\left(\omega_{g}\right)$ of $T_{A}$ (respectively, $T_{B}$ ).

\section{THE PROOF}

We require the following fact, which is certainly well-known (compare [2], $\S 6.7$ for the case of a simple closed curve).

Lemma 4.1. Let $C=c_{1} \cup \ldots \cup c_{n}$ be a multicurve, $T_{C}=T_{c_{1}} \circ \ldots \circ T_{c_{n}}$ the corresponding multitwist and $[\mu] \in \mathbb{P} \mathcal{M L}(S)$ with $i\left(\mu, c_{j}\right) \neq 0$ for each $1 \leq j \leq n$. Then

$$
\lim _{k \rightarrow \infty} T_{C}^{k}([\mu])=\left[i\left(\mu, c_{1}\right) c_{1}+\ldots+i\left(\mu, c_{n}\right) c_{n}\right]
$$

Proof. We assume for simplicity that no two components of $C$ are isotopic. If this is not the case, collect together isotopic components and replace the composition of the associated Dehn twists by a power of a single twist in one of the curves - a composition of Dehn twists in pairwise isotopic curves is isotopic to a power of a twist in one of the curves.

In what follows we use several facts from the theory of train tracks. We refer the reader to 13 for more on the concepts we use here.

There exists a birecurrent train track $\tau$ carrying $\mu$ so that in a neighborhood of each component $c_{j}$, the local picture is as in Figure 2 on the left (this is one of the "standard model" train tracks of [13] for an appropriate choice of basis multicurves, depending on $C$ and $\mu$ ). Near $c_{j}$ the weights on the branches of $\tau$ determined by $\mu$ are $x_{j}=i\left(\mu, c_{j}\right), y_{j}$, and $z_{j}=x_{j}+y_{j}$ (which is the weight on the branch not labeled in the figure). We also see that each $c_{j}$ is carried by $\tau$ with the corresponding weights given by $\tilde{x}_{j}=0, \tilde{y}_{j}=\tilde{z}_{j}=1$ and all other weights zero.

Note that $T_{C}(\tau)$ is carried by $\tau$, and so $T_{C}(\mu)$ is also carried by $\tau$. The change in weights from $\mu$ to $T_{C}(\mu)$ is described on the right of Figure 2. Observe that all weights stay the same, except each of the $y_{j}$ and $z_{j}$ which both have $x_{j}$ added to them. It follows that

$$
\lim _{k \rightarrow \infty} \frac{1}{k} T_{C}^{k}(\mu)=x_{1} c_{1}+\ldots+x_{n} c_{n}=i\left(\mu, c_{1}\right) c_{1}+\ldots+i\left(\mu, c_{n}\right) c_{n} .
$$

We are now prepared to prove the main theorem. 

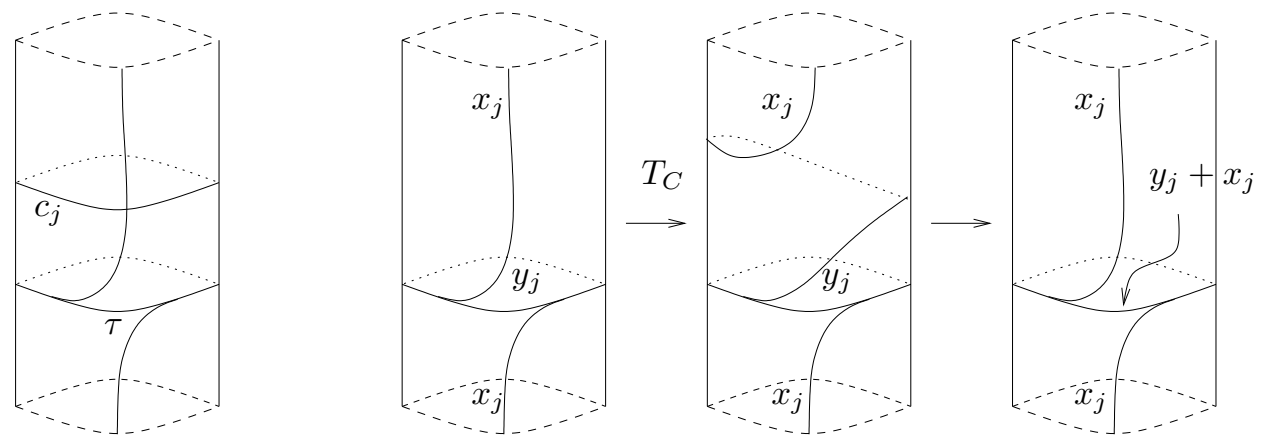

FiguRE 2. Left: The train track near $c_{j}$. Right: Dehn twisting in $C$ (first arrow) and how $\tau$ carries $T_{C}(\tau)$ (second arrow).

Proof of Theorem 1.2, We begin by defining the set $Y$. First, write

$$
\left[\nu_{B}\right]=\left[w_{1} b_{1}+\ldots+w_{g} b_{g}\right],
$$

where $w_{1}, \ldots, w_{g} \in \mathbb{R}_{+}$are determined by $\omega_{g}$-these are the heights of the cylinders as described above. View $X \subset \mathbb{P} \mathcal{M L}(S-A) \subset \mathbb{P} \mathcal{M L}(S)$ and set

$$
Y=\left\{[\mu] \in X \mid \frac{i\left(\mu, b_{i}\right)}{i\left(\mu, b_{j}\right)}=\frac{w_{i}}{w_{j}} \text { for all } 1 \leq i, j \leq g\right\} .
$$

The set $Y$ is obtained from $X$ by imposing certain relations on intersection numbers. Observing how the components of $B$ intersect $S-A$ (see Figure 1), one easily verifies that $Y$ is a positive codimension subset of $X$.

Now let $\left[\mu_{s}\right]$ denote the stable lamination of $h$ and assume $\left[\mu_{s}\right] \notin Y$. We suppose that there exists a Cannon-Thurston map $f: \partial \mathcal{G}\left(\omega_{g}, h, n\right) \rightarrow \mathbb{P} \mathcal{M L}(S)$ and arrive at a contradiction, provided $n$ is sufficiently large.

First observe that since $\Lambda_{\mathcal{G}\left(\omega_{g}, h, n\right)}$ is the minimal closed $\mathcal{G}\left(\omega_{g}, h, n\right)$-invariant set, $f\left(\partial \mathcal{G}\left(\omega_{g}, h, n\right)\right)=\Lambda_{\mathcal{G}\left(\omega_{g}, h, n\right)}$.

As described above, $\left(T_{A}^{2(2 g+1)} \sigma\right) \in \mathcal{G}\left(\omega_{g}, h, n\right)$ and hence all its $\mathcal{G}\left(\omega_{g}, h, n\right)$ conjugates also lie in $\mathcal{G}\left(\omega_{g}, h, n\right)$. In particular, $\left(T_{B}^{2(2 g+1)} \sigma\right) \in \mathcal{G}\left(\omega_{g}, h, n\right)$. Since this is the fundamental group of a closed surface, $\left(T_{B}^{2(2 g+1)} \sigma\right)$ has exactly two fixed points $x^{ \pm} \in \partial \mathcal{G}\left(\omega_{g}, h, n\right): x^{+}$the attracting fixed point and $x^{-}$the repelling fixed point.

Since $G\left(\omega_{g}\right)<\mathcal{G}\left(\omega_{g}, h, n\right)$, we have

$$
\mathbb{P} \mathcal{M L}\left(\omega_{g}\right)=\Lambda_{G\left(\omega_{g}\right)} \subset \Lambda_{\mathcal{G}\left(\omega_{g}, h, n\right)} \subset \mathbb{P} \mathcal{M L}(S) .
$$

Claim. $f\left(x^{ \pm}\right)=\left[\nu_{B}\right]$.

Proof. By considering the action of the parabolic $D\left(T_{B}^{2(2 g+1)} \sigma\right)$ on $\partial \mathbb{H}_{\omega_{g}}$ and applying the $G\left(\omega_{g}\right)$-equivariant homeomorphism $\partial \mathbb{H}_{\omega_{g}} \rightarrow \mathbb{P} \mathcal{M L}\left(\omega_{g}\right)$, iterating $\left(T_{B}^{2(2 g+1)} \sigma\right)$ on $\left[\nu_{A}\right] \in \mathbb{P} \mathcal{M L}\left(\omega_{g}\right)$ we see

$$
\lim _{k \rightarrow \pm \infty}\left(T_{B}^{2(2 g+1)} \sigma\right)^{k}\left(\left[\nu_{A}\right]\right)=\left[\nu_{B}\right] .
$$


If we let $y \in \partial \mathcal{G}\left(\omega_{g}, h, n\right)$ be an element with $f(y)=\left[\nu_{A}\right]$, then by continuity and $\mathcal{G}\left(\omega_{g}, h, n\right)$-equivariance of $f$ we obtain

$$
\begin{aligned}
{\left[\nu_{B}\right] } & =\lim _{k \rightarrow \pm \infty}\left(T_{B}^{2(2 g+1)} \sigma\right)^{k}(f(y))=\lim _{k \rightarrow \pm \infty} f\left(\left(T_{B}^{2(2 g+1)} \sigma\right)^{k}(y)\right) \\
& =f\left(\lim _{k \rightarrow \pm \infty}\left(T_{A}^{2(2 g+1)} \sigma\right)^{k}(y)\right)=f\left(x^{ \pm}\right),
\end{aligned}
$$

proving the claim.

Since $h^{n} G\left(\omega_{g}\right) h^{-n}<\mathcal{G}\left(\omega_{g}, h, n\right)$ and

$$
h^{n}\left(\Lambda_{G\left(\omega_{g}\right)}\right)=\Lambda_{h^{n} G\left(\omega_{g}\right) h^{-n}} \subset \Lambda_{\mathcal{G}\left(\omega_{g}, h, n\right)},
$$

it follows that $h^{n}\left(\left[\nu_{B}\right]\right) \in \Lambda_{\mathcal{G}\left(\omega_{g}, h, n\right)}=f\left(\partial \mathcal{G}\left(\omega_{g}, h, n\right)\right)$. Therefore, there exists $z \in$ $\partial \mathcal{G}\left(\omega_{g}, h, n\right)$ with $f(z)=h^{n}\left(\left[\nu_{B}\right]\right)$, and so again by the continuity and $\mathcal{G}\left(\omega_{g}, h, n\right)-$ equivariance of $f$ as well as the claim above,

$$
\begin{aligned}
{\left[\nu_{B}\right]=f\left(x^{+}\right) } & =f\left(\lim _{k \rightarrow \infty}\left(T_{B}^{2(2 g+1)} \sigma\right)^{k}(z)\right)=\lim _{k \rightarrow \infty}\left(T_{B}^{2(2 g+1)} \sigma\right)^{k}(f(z)) \\
& =\lim _{k \rightarrow \infty}\left(T_{B}^{2(2 g+1)} \sigma\right)^{k}\left(h^{n}\left(\left[\nu_{B}\right]\right)\right)=\lim _{k \rightarrow \infty} T_{B}^{2 k(2 g+1)}\left(h^{n}\left(\left[\nu_{B}\right]\right)\right),
\end{aligned}
$$

where the last equality follows from the fact that $\sigma$ commutes with both $h$ and $T_{B}$, and $\sigma$ fixes $\left[\nu_{B}\right]$. Appealing to Lemma 4.1 this implies

$$
\left[\nu_{B}\right]=\left[i\left(h^{n}\left(\nu_{B}\right), b_{1}\right) b_{1}+\ldots+i\left(h^{n}\left(\nu_{B}\right), b_{g}\right) b_{g}\right] .
$$

Combining this with (4.1) we see that for all $1 \leq i, j \leq g$,

$$
\frac{i\left(h^{n}\left(\nu_{B}\right), b_{i}\right)}{i\left(h^{n}\left(\nu_{B}\right), b_{j}\right)}=\frac{w_{i}}{w_{j}} .
$$

Since $\mu_{s}$ fills $S-A$, we have $i\left(\nu_{B}, \mu_{s}\right) \neq 0$, and so by Theorem A1 of [4,

$$
\lim _{n \rightarrow \infty} h^{n}\left(\left[\nu_{B}\right]\right)=\left[\mu_{s}\right]
$$

Therefore, for every $1 \leq i, j \leq g$,

$$
\lim _{n \rightarrow \infty} \frac{i\left(h^{n}\left(\nu_{B}\right), b_{i}\right)}{i\left(h^{n}\left(\nu_{B}\right), b_{j}\right)}=\frac{i\left(\mu_{s}, b_{i}\right)}{i\left(\mu_{s}, b_{j}\right)} .
$$

Since $\left[\mu_{s}\right] \notin Y$, there exist indices $1 \leq i, j \leq g$ so that

$$
\frac{i\left(\mu_{s}, b_{i}\right)}{i\left(\mu_{s}, b_{j}\right)} \neq \frac{w_{i}}{w_{j}} .
$$

Thus by (4.3), for all sufficiently large $n$,

$$
\frac{i\left(h^{n}\left(\nu_{B}\right), b_{i}\right)}{i\left(h^{n}\left(\nu_{B}\right), b_{j}\right)} \neq \frac{w_{i}}{w_{j}}
$$

which contradicts (4.2). This completes the proof.

\section{ACKNOWLEDGEMENTS}

Thanks to Richard Kent and Sergio Fenley for their interest and helpful suggestions and conversations. 


\section{REFERENCES}

1. Danny Calegari, Real places and torus bundles, Geom. Dedicata 118 (2006), 209-227. MR 2239457

2. A. Fathi, F. Laudenbach, and V. Poénaru, Travaux de Thurston sur les surfaces, Société Mathématique de France, Paris, 1991, Séminaire Orsay; reprint of Travaux de Thurston sur les surfaces, Soc. Math. France, Paris, 1979, Astérisque No. 66-67 (1991). MR 1134426 (92g:57001)

3. W. J. Floyd, Group completions and limit sets of Kleinian groups, Invent. Math. 57 (1980), no. 3, 205-218. MR.568933 (81e:57002)

4. N. V. Ivanov, Subgroups of Teichmüller modular groups, Translations of Mathematical Monographs, vol. 115, American Mathematical Society, Providence, RI, 1992; translated from the Russian by E. J. F. Primrose and revised by the author. MR.1195787 (93k:57031)

5. Richard P. Kent IV and Christopher J. Leininger, Subgroups of mapping class groups from the geometrical viewpoint, In the tradition of Ahlfors-Bers. IV, Contemp. Math., vol. 432, Amer. Math. Soc., Providence, RI, 2007, pp. 119-141. MR2342811

6. C. J. Leininger, Graphs of Veech groups, work in progress.

7. On groups generated by two positive multi-twists: Teichmüller curves and Lehmer's number, Geom. Topol. 8 (2004), 1301-1359 (electronic). MR2119298 (2005j:57002)

8. C. J. Leininger and A. W. Reid, A combination theorem for Veech subgroups of the mapping class group, Geom. Funct. Anal. 16 (2006), no. 2, 403-436. MR2231468 (2007d:57002)

9. B. Maskit, Kleinian groups, Grundlehren der Mathematischen Wissenschaften [Fundamental Principles of Mathematical Sciences], vol. 287, Springer-Verlag, Berlin, 1988. MR959135 (90a:30132)

10. H. A. Masur, Two boundaries of Teichmüller space, Duke Math. J. 49 (1982), no. 1, 183-190. MR650376 (83k:32035)

11. J. McCarthy and A. Papadopoulos, Dynamics on Thurston's sphere of projective measured foliations, Comment. Math. Helv. 64 (1989), no. 1, 133-166. MR.982564 (90e:57054)

12. Lee Mosher, Problems in the geometry of surface group extensions, Problems on mapping class groups and related topics, Proc. Sympos. Pure Math., vol. 74, Amer. Math. Soc., Providence, RI, 2006, pp. 245-256. MR2264544

13. R. C. Penner and J. L. Harer, Combinatorics of train tracks, Annals of Mathematics Studies, vol. 125, Princeton University Press, Princeton, NJ, 1992. MR.1144770 (94b:57018)

14. W. A. Veech, Teichmüller curves in moduli space, Eisenstein series and an application to triangular billiards, Invent. Math. 97 (1989), no. 3, 553-583. MR.1005006 (91h:58083a)

15. __ Erratum: "Teichmüller curves in moduli space, Eisenstein series and an application to triangular billiards", Invent. Math. 103 (1991), no. 2, 447. MR1085115 (91h:58083b)

Department of Mathematics, University of Illinois at Urbana-Champaign, 1409 W. Green Street, Urbana, Illinois 61801

E-mail address: clein@math.uiuc.edu 